\title{
Chronic Subdural Hematoma Following Dural Metastasis of Gastric Cancer: Measurement of Pre- and Postoperative Cerebral Blood Flow With $\mathrm{N}$-isopropyl-p-[ $\left.{ }^{123} I\right]$ iodoamphetamine
}

\author{
-Case Report-
}

\author{
Koichi FUKINO, Takeshi TERAO, Toyoyuki KOJIMA, Koji ADACHI, \\ and Akira TERAMOTO
}

Department of Neurosurgery, Nippon Medical School, Tokyo

\begin{abstract}
A 58-year-old female with gastric cancer presented with left chronic subdural hematoma (CSH) without history of head injury. Magnetic resonance imaging revealed left $\mathrm{CSH}$ with atypical findings such as abnormal dural enhancement and swelling of the left cerebral hemisphere. One month after gastrectomy, motor aphasia and right hemiparesis developed. Irrigation of the left CSH was performed. The hematoma was abnormally mucinous and became solid immediately after irrigation. Histological examination showed that adenocarcinoma cells had metastasized to the dura mater and the outer membrane of the hematoma. The preoperative cerebral blood flow (CBF) in the affected cerebral hemisphere, measured by single photon emission computed tomography using $\mathrm{N}$-isopropyl-p-[123I]iodoamphetamine, was much higher than that in the opposite hemisphere, whereas the postoperative CBF was almost equal in both hemispheres. Subdural hematomas secondary to dural metastases of extraneuronal malignancies are rare, and are usually the chronic type. Measurement of the pre- and postoperative CBF in the present patient with CSH following dural metastasis of the malignant tumor showed that preoperative hyperemia in the affected hemisphere may result from dilation of the cerebral vessels caused by the effects of the CSH.
\end{abstract}

Key words: magnetic resonance imaging, N-isopropyl-p-[123I]iodoamphetamine single photon emission computed tomography, chronic subdural hematoma, dural metastasis, malignant tumor,

cerebral blood flow

\section{Introduction}

Non-traumatic subdural hematomas have various causes, including vascular disorders, coagulopathies, intracranial hypotension, meningitis, and dural metastases of malignant tumors. ${ }^{2)}$ Subdural hematomas secondary to dural metastases of extraneuronal malignancies are rare, and are usually the chronic type. ${ }^{1)}$ The common primary sites include the stomach, breast, lung, and prostate. ${ }^{12)}$ Cerebral blood flow (CBF) has been examined in the patients with non-tumoral chronic subdural hematomas (CSHs), but not in patients with CSHs associ- ated with meningeal carcinomatosis.

The present study measured both preoperative and postoperative $\mathrm{CBF}$ by $\mathrm{N}$-isopropyl-p-[ $\left.{ }^{123} \mathrm{I}\right]$ iodoamphetamine single photon emission computed tomography (IMP-SPECT) in a 58-year-old female with CSH secondary to dural metastasis from gastric cancer.

\section{Case Report}

A 58-year-old female underwent total gastrectomy because of gastric cancer (type 4). She suffered from loss of appetite and headache. Magnetic resonance

Received July 10, 2003; Accepted June 18, 2004

Author's present address: K. Fukino, M.D., Division of Human Cancer Genetics, The Ohio State University Tzagournis Medical Research Facility, Columbus, Ohio, U.S.A. 

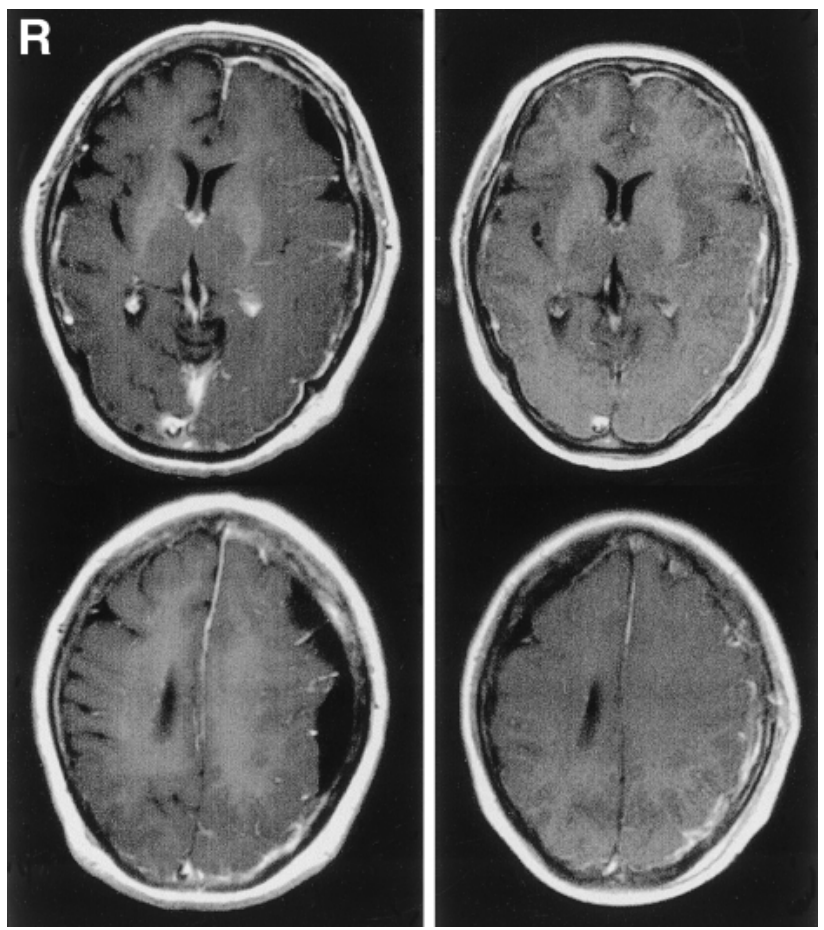

Fig. 1 Preoperative

$T_{1}$-weighted

magnetic resonance (MR) images with gadolinium demonstrating left chronic subdural hematoma associated with abnormal dural enhancement, manifestation of the vessels in the left hemisphere, and swelling of the left hemisphere (left column). Postoperative $T_{1}$-weighted MR images with gadolinium showing that the subdural hematoma had completely disappeared and the vessels in the left hemisphere were less visible, whereas the dural enhancement persisted (right column).

(MR) imaging with contrast enhancement revealed left $\mathrm{CSH}$ with atypical findings, such as abnormal enhancement of the dura mater, manifestation of the vessels in the left cerebral hemisphere, and swelling of the involved hemisphere (Fig. 1 left column). These findings corresponded to group 2 of the MR imaging classification of meningeal carcinomatosis. ${ }^{15)}$ One month after gastrectomy, motor aphasia and right hemiparesis occurred in the night. Computed tomography (CT) showed slight increase of the subdural hematoma and deterioration of the swelling of the left cerebral white matter. IMPSPECT of the head, performed 6 days after the onset, showed that the CBF of the left cerebral hemisphere was much higher than that of the right (Fig. 2 upper row). The CBF of the ipsilateral and contralateral cerebellar hemispheres were similar.
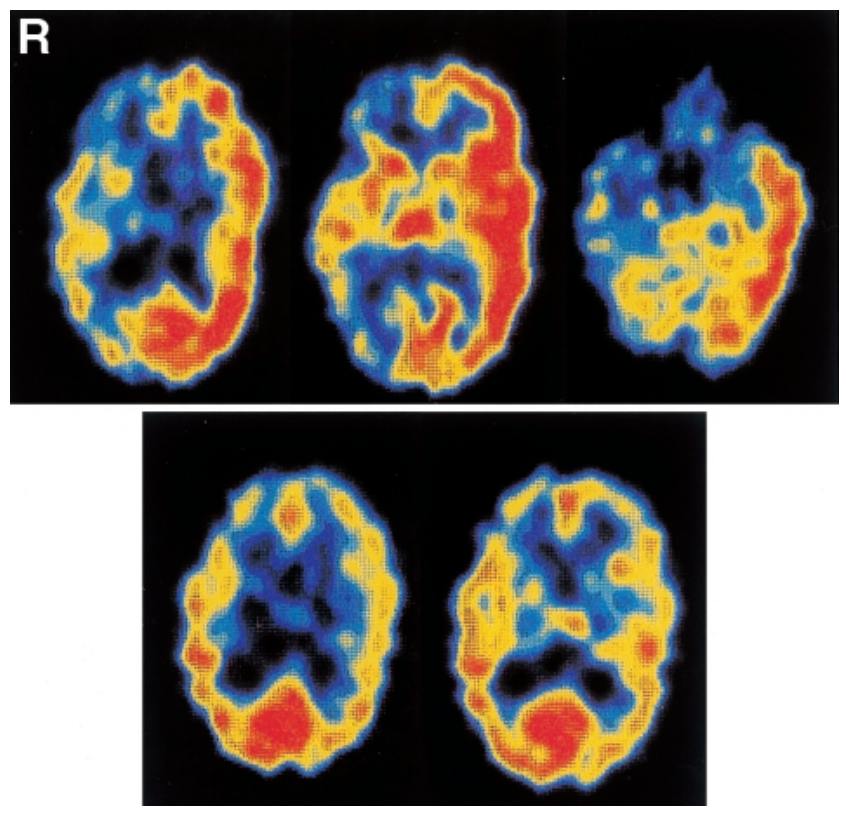

Fig. 2 Preoperative N-isopropyl-p-[ $\left.{ }^{123} \mathrm{I}\right]$ iodoamphetamine single photon emission computed tomography (IMP-SPECT) scans revealing that the cerebral blood flow (CBF) was much higher in the left cerebral hemisphere than in the right (upper row). Postoperative IMP-SPECT scans showing that the CBF in the left hemisphere was almost equal to that in the right (lower row). The preoperative scans showed the CBF of the contralateral cerebellar hemisphere was not elevated compared to that of the ipsilateral hemisphere, suggesting absence of remote effect.
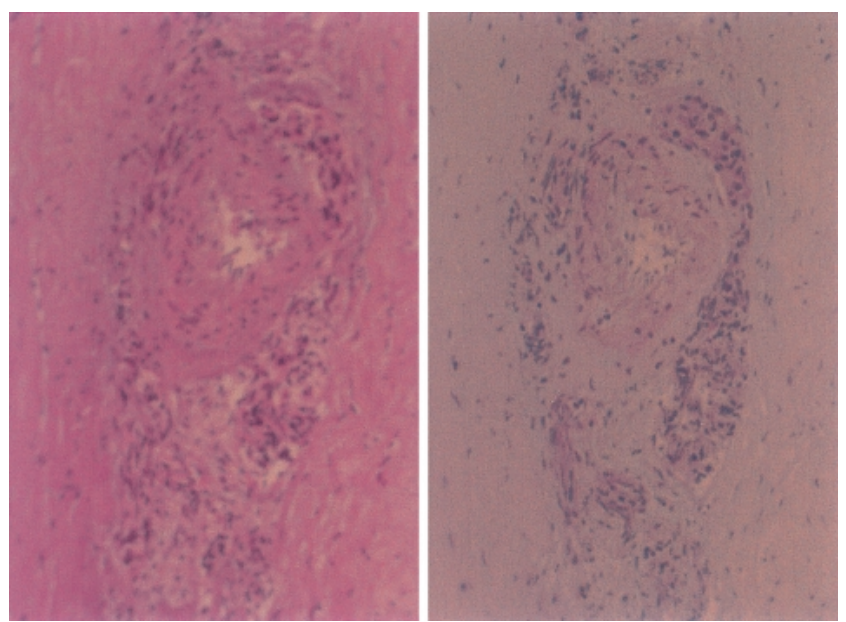

Fig. 3 Photomicrographs of the dura mater showing perivascular infiltration of atypical cells (left: hematoxylin-eosin stain, $\times 20$ ), containing cytoplasm positive for periodic acid-Schiff stain (right: $\times 20$ ). 
Administration of mannitol only transiently and slightly improved her aphasia and right hemiparesis. One and a half months after gastrectomy, irrigation of the CSH was performed together with biopsy of the dura mater and the outer membrane of the hematoma. The dura mater was rather thickened, and the hematoma was abnormally mucinous and became solid immediately after irrigation. Histological examination of the dura mater and the outer membrane revealed perivascular infiltration of atypical cells, which contained cytoplasm positive for periodic acid-Schiff stain (Fig. 3). These histological findings were compatible with dural metastasis of adenocarcinoma.

Immediately after the operation, her headache and nausea disappeared, and aphasia and motor weakness of the right limbs improved. Postoperative head CT showed that the CSH had almost completely disappeared. The vessels in the left hemisphere, prominent on the preoperative MR image, had become inconspicuous on the postoperative MR image, whereas the dura mater was still enhanced as strongly as before (Fig. 1 right column). Postoperative IMP-SPECT showed that the CBF of the left and right cerebral hemispheres had become almost equal (Fig. 2 lower row).

Her general condition and neurological findings transiently improved. However, she soon developed visual disturbance, probably due to thickening of the dura mater in the optic canals, and she died of disseminated intravascular coagulation one and a half months after irrigation of the CSH. Autopsy was not permitted.

\section{Discussion}

Several mechanisms of CSH formation associated with dural metastasis have been postulated. Obstruction of the dural veins by tumor cells would cause dilation of the capillaries in the inner areolar layer, followed by rupture and subdural hemorrhage. ${ }^{13)}$ Hyperplasia of the inner areolar layer is an angiodesmoplastic response to the tumor invading the meninges. ${ }^{10)}$ Therefore, hematoma could result from separation and hemorrhage into the highly vascular abnormal areolar layer of the dura mater. ${ }^{1)}$ The combined effect of metastases in dural vessels with a coagulation defect could lead to large subdural hematomas. ${ }^{1)}$ Secretion by the mucin-producing carcinoma was the proposed pathogenic mechanism in a case of dural metastasis from an adenocarcinoma of the pancreas. ${ }^{3)}$ The abnormally mucinous hematoma in our case suggests that secretion by the carcinoma cells could be a pathogenic mechanism.

$\mathrm{CBF}$ is not necessarily correlated with the type of lesion, but can be affected by arteriovenous shunts, blood-brain barrier dysfunction, and blood vessels lacking autoregulation in or around the lesion. ${ }^{9}$ CBF depression occurs in the hemisphere under $\mathrm{CSH}$, but the reported degree and/or the localization of the CBF depression differ. ${ }^{5-7,11,14)}$ In the present case, IMP-SPECT showed much higher preoperative uptake on the lesion side than that on the non-lesion side, whereas the abnormally higher uptake diminished after irrigation of the CSH. A previous case of adenocarcinoma of the stomach also demonstrated higher IMP uptake. ${ }^{4)}$ However, the higher uptake of IMP in the present case could not be attributed to the factors of adenocarcinoma cells, because simple irrigation of the hematoma might have little effect on the number and the metabolic activities of tumor cells. The vessels under subdural hematomas are pathologically dilated in patients with head injury, and the dilation is reduced after removal of the hematomas. ${ }^{8)}$ In the present case, the preoperative increased uptake on IMP-SPECT may be due to dilation of the vessels, possibly caused by the reduced venous return due to compression to the cerebral veins by the CSH. Otherwise, mechanical compression or chemical stimulation by the $\mathrm{CSH}$ may have damaged the autoregulation of the vessels. Irrigation of the CSH might remove or decrease such harmful effects. Preoperative SPECT showed no remote-effect signs suggesting the metabolic coupling accompanying seizures. The hyperemia together with compression by the hematoma might have caused the swelling of the left hemisphere.

The etiology of the neurological symptoms was unclear. Compression by the $\mathrm{CSH}$, hyperemia associated with brain swelling, and seizure are all possible causes. The rather long duration of the neurological deficits and the sudden improvement immediately after irrigation tend to exclude seizure. The combination of compression by the $\mathrm{CSH}$ and deterioration of brain swelling is the most likely cause, because the neurological deficits were transiently improved by the mannitol administration and irrigation of the $\mathrm{CSH}$.

Measurement of CBF in the present patient with $\mathrm{CSH}$ associated with dural metastasis of malignant tumor found abnormally high preoperative uptake in the affected hemisphere which normalized after irrigation of the CSH. Patients with dural metastases of malignant tumors may experience transient improvement of the neurological conditions by irrigation of the hematoma, but many of them will have very poor outcomes. Histological examination of the dura mater and the outer membrane are very important in patients with $\mathrm{CSH}$ and histories of malignant tumors. 


\section{References}

1) Ambiavagar PC, Sher J: Subdural hematoma secondary to metastatic neoplasm: report of two cases and review of the literature. Cancer 42: 2015-2018, 1978

2) Bergmann M, Puskas Z, Kuchelmeister K: Subdural hematoma due to dural metastasis: case report and review of the literature. Clin Neurol Neurosurg 94: 235-240, 1992

3) Castleman B, Scully RE, McNeely BU: Presentation of case 12 - 1972. N Engl J Med 286: 650-656, 1972

4) Hirano $T$, Fukuda $T$, Mogi $Y$, Kawashima M, Tatezawa T, Kobayashi I, Ogawa T: Adenocarcinoma mimicking primary gastric lymphoma demonstrated by I-123 IMP scintigraphy. Clin Nucl Med 22: 628-631, 1997

5) Horinaka N, Yasumoto Y, Kumami K, Matsumura K: Evaluation of regional cerebral blood flow in chronic subdural hematoma. Keio J Med 49 Suppl 1: A156-158, 2000

6) Isaka $Y$, Imaizumi $M$, Itoi $Y$, Ashida $K$, Horibe $K$ : Cerebral blood flow imaging with technetium-99mHMPAO SPECT in a patient with chronic subdural hematoma: relationship with neuropsychological test. J Nucl Med 33: 246-248, 1992

7) Ishikawa T, Kawamura S, Hadeishi H, Suzuki A, Yasui N, Uemura K: Cerebral blood flow and oxygen metabolism in hemiparetic patients with chronic subdural hematoma; quantitative evaluation using positron emission tomography. Surg Neurol 43: 130-137, 1995

8) Kuhl DE, Alavi A, Hoffman EJ, Phelps ME, Zimmerman RA, Obrist WD, Bruce DA, Greenberg JH, Uzzell B: Local cerebral blood volume in head-injured patients. Determination by emission computed tomography of ${ }^{99 m}$ Tc-labeled red cells. J Neurosurg
52: 309-320, 1980

9) LaFrance ND, Wagner HN, Whitehouse P, Corley E, Duelfer T: Decreased accumulation of isopropyliodoamphetamine (I-123) in brain tumor. J Nucl Med 22: 1081-1083, 1081

10) Leech RW, Welch FT, Ojemann GA: Subdural hematoma secondary to metastatic dural carcinomatosis: case report. J Neurosurg 41: 610-613, 1974

11) Okuyama T, Saito K, Fukuyama K, Yamamoto $K$, Morimoto M, Aburano T: [Clinical study of cerebral blood flow in unilateral chronic subdural hematoma measured by ${ }^{99 m}$ Tc-HMPAO SPECT]. No To Shinkei 52: 141-147, 2000 (Jpn, with Eng abstract)

12) Otsuka A, Asakura K, Takahashi K, Tasaki T, Okada K, Suzuki Y: [Nontraumatic chronic subdural hematoma due to dural metastases of breast cancer: case report]. No Shinkei Geka 13: 999-1004, 1985 (Jpn, with Eng abstract)

13) Russel DS, Cairns H: Subdural false membrane or hematoma (pachymeningitis interna hemorrhagica) in carcinomatosis and sarcomatosis of the dura mater. Brain 57: 32-48, 1934

14) Toyama H, Ichise M, Schacter BI, Kirsh JC: 'Reverse crescent pattern' on SPECT brain perfusion scan in chronic subdural hematoma. Clin Nucl Med 17: 473-476, 1992

15) Watanabe $M$, Tanaka $R$, Takeda N: Correlation of MRI and clinical features in meningeal carcinomatosis. Neuroradiology 35: 512-515, 1993

Address reprint requests to: K. Fukino, M.D., Department of Neurosurgery, Nippon Medical School, 1-1-5 Sendagi, Bunkyo-ku, Tokyo 113-8602, Japan. e-mail: koichifukino@aol.com 